\title{
Análise da acessibilidade nos MOOCs das universidades federais do Brasil em conformidade com os requisitos do W3C e eMAG
}

\author{
Pierre Correa Martin - PPGMAE/UNIPAMPA - pierresi@gmail.com \\ Sandra Dutra Piovesan - PPGMAE/UNIPAMPA - sanpiovesan@ gmail.com
}

\begin{abstract}
Resumo: Os Massive Open Online Course - MOOC são cursos abertos, gratuitos e online, que através de recursos tecnológicos possibilitam o processo de ensino-aprendizagem em larga escala. Nessa pesquisa foram identificadas sessenta e três universidades federais do Brasil, as quais foram consultadas, através da Lei de Acesso à Informação, quanto à oferta de MOOCs para a sociedade. Estes foram avaliados de forma automática, em sites específicos para esse fim, quanto ao atendimento das recomendações de acessibilidade do World Wide Web Consortium W3C e do Modelo de Acessibilidade em Governo Eletrônico - eMAG. Além disso, foi realizada uma análise manual nos MOOCs, visando identificar a possibilidade de uso em dispositivos móveis e a presença dos requisitos do eMAG para conteúdo multimídia, mais especificamente os vídeos. Os resultados apontaram que embora alguns itens de acessibilidade sejam atendidos, ainda há necessidade de melhorias nas plataformas e nos conteúdos disponibilizados.
\end{abstract}

Palavras-chave: MOOC, acessibilidade, W3C, eMAG.

\section{MOOCs accessibility analysis of Brazilian federal universities in accordance with $\mathrm{W} 3 \mathrm{C}$ and eMAG requirements}

\begin{abstract}
Massive Open Online Course - MOOC is a free, open online course that through technological resources enables the process of teaching and learning on a large scale. In this research were identified sixty-three federal universities in Brazil, which were consulted, through the Law on Access to Information, regarding the offer of MOOCs to society. These were automatically evaluated, on specific sites for this purpose, regarding compliance with the accessibility recommendations of the World Wide Web Consortium - W3C and the eMAG Accessibility Model. In addition, a manual analysis of the MOOCs was performed to identify the possibility of use on mobile devices and the presence of eMAG requirements for multimedia content, specifically videos. The results pointed out that although some accessibility items are met, there is still a need for improvements in the platforms and content available.
\end{abstract}

Keywords: MOOC, accessibility, W3C, eMAG.

\section{Introdução}

Com os avanços tecnológicos e a expansão da internet nas últimas décadas, surgiu uma sociedade voltada para a informação e para o uso de recursos multimídias, os quais, promovem agilidade no uso das informações e minimizam barreiras, auxiliando no processo de ensino-aprendizagem (Candeias, Carvalho, 2016). À partir disso, instituições públicas e privadas passaram a ofertar de diferentes formas os conteúdos produzidos, visando garantir a aquisição e a fixação dos conhecimentos por parte dos alunos. Uma das tecnologias utilizadas é o Massive Open Online Course MOOC, plataforma de cursos gratuitos oferecidos para a sociedade, com o objetivo de atender uma grande quantidade de indivíduos (Gonçalves, 2013). 
Para garantir que os MOOCs atendam toda a população, faz-se necessário que os mesmos tenham requisitos de acessibilidade. Nesse sentido, Ferraz (2017, p. 4) diz que "a acessibilidade é fundamental para garantir o acesso para todas as pessoas, especialmente para pessoas com deficiência, que muitas vezes acabam impedidas de atingir determinados conteúdos por certas barreiras de acesso". Visando garantir a acessibilidade web, o Word Wide Web Consortium - W3C Brasil e o Modelo de Acessibilidade em Governo Eletrônico - eMAG definem padrões para implementação de conteúdos online.

Dessa forma, o objetivo geral desta pesquisa é identificar os MOOCs disponibilizados pelas universidades federais do país e avaliar a acessibilidade que estes oferecem, visando sanar a incerteza prévia quanto ao grau de inclusão ofertado nos cursos, fato este que justifica o estudo. Para o alcance desse objetivo, foram elencados como objetivos específicos: a análise automática da acessibilidade da plataforma de acordo com o W3C e eMAG, a possibilidade de utilização da plataforma em dispositivos móveis e o atendimento aos requisitos propostos pelo eMAG para conteúdos multimídia, mais especificamente, os vídeos.

A apresentação do estudo divide-se no artigo em introdução e referencial teórico, metodologia, resultados e conclusão. A introdução ao tema, contextualizou e apresentou objetivos e justificativas para a realização da pesquisa. O referencial teórico e a metodologia utilizados para a confecção do artigo, aprofundaram os conceitos e expuseram dados e legislações vigentes bem como, descreveram os passos realizados para a obtenção das informações. Por fim, os resultados e as conclusões apresentaram os dados obtidos através do trabalho realizado.

\section{Acessibilidade e MOOC}

Os conceitos, a origem e a importância dos assuntos abordados na pesquisa são apresentados à seguir:

\subsection{Acessibilidade}

A acessibilidade é um tema recorrente em ambientes que buscam identificar alternativas para a inclusão de todas as pessoas e em discussões relacionadas a políticas públicas. Estas, apresentam como resultados a Lei $\mathrm{n}^{\circ} 10.048$, de 8 de novembro de 2000, que regulamenta o acesso prioritário a pessoas específicas e a Lei $\mathrm{n}^{\circ} 10.098$, de 19 de dezembro de 2000, conhecida como "Lei da Acessibilidade", que promove a acessibilidade para pessoas com deficiência ou mobilidade reduzida (Brasil, 2000).

Além disso, em 6 de julho de 2015, foi instituída a Lei Brasileira de Inclusão da Pessoa com Deficiência, no 13.146, destinada a "assegurar e a promover, em condições de igualdade, o exercício dos direitos e das liberdades fundamentais por pessoa com deficiência, visando à sua inclusão social e cidadania". Dessa forma, é possível perceber iniciativas com o objetivo de atender da melhor forma o grande número de cidadãos brasileiros que apresentam algum tipo de deficiência.

Segundo os dados do Instituto Brasileiro de Geografia e Estatística - IBGE, apurados no Censo realizado em 2010, 45,6 milhões de brasileiros apresentam algum tipo de deficiência, o que representa aproximadamente $24 \%$ da população. Esse número cai para $6,7 \%$ se for considerado somente as pessoas que apresentam deficiência mental, intelectual ou que possuem grande ou total dificuldade para enxergar, ouvir, caminhar ou subir degraus. Essas, estão representadas conforme gráfico abaixo (IBGE, 2012). 


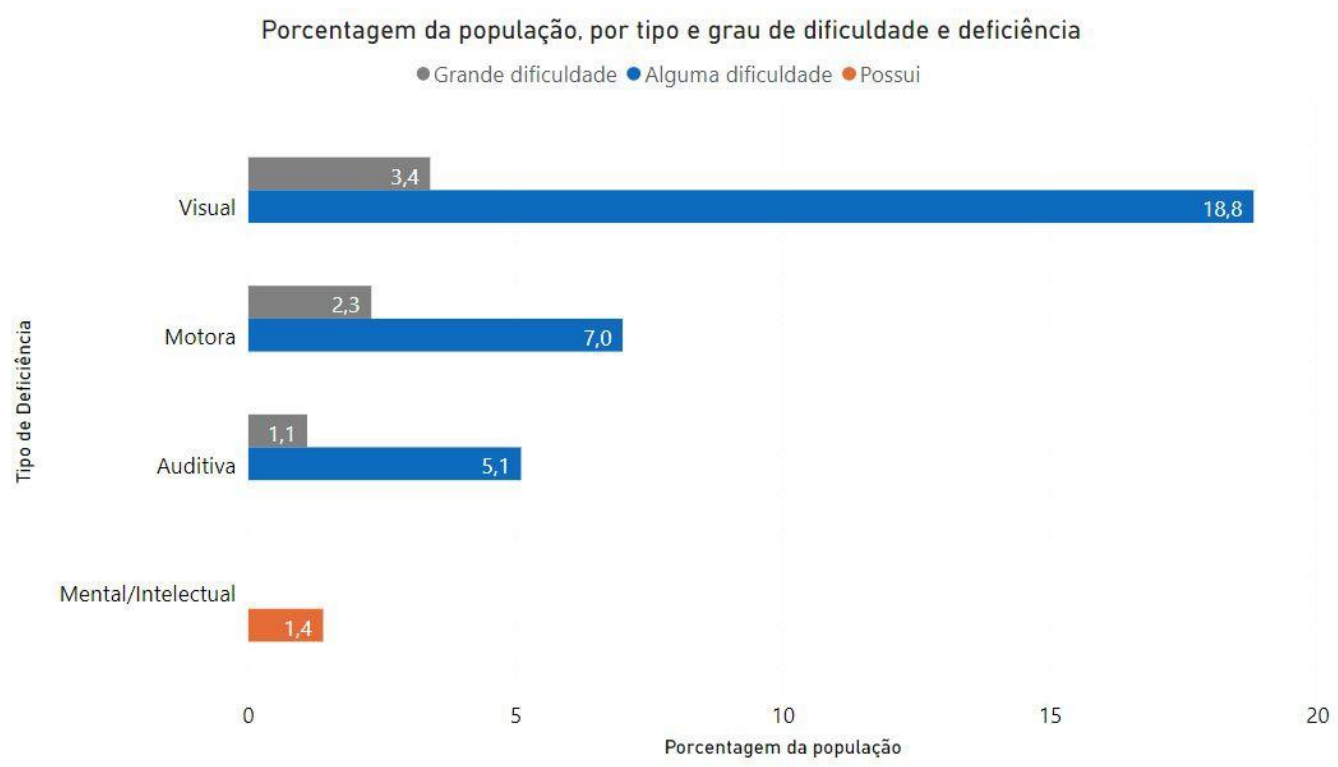

Figura 1 - gráfico da porcentagem de população por tipo e grau de deficiência

Considerando respectivamente os valores para alguma dificuldade e grande dificuldades, o gráfico acima apresenta os índices de 18,8\% e 3,4\% para deficiência visual, $7,0 \%$ e $2,3 \%$ para deficiência motora e $5,1 \%$ e $1,1 \%$ para deficiência auditiva, além de 1,4\% para deficiência mental/intelectual (IBGE, 2012).

\subsection{Acessibilidade na WEB}

A acessibilidade na web tornou-se fundamental garantir o acesso universal, pensando nisso, órgãos públicos de execução e controle, passaram a atentar para a implementação de requisitos de acessibilidade em suas plataformas através do W3C e eMAG. Esse, criou as Diretrizes de Acessibilidade para Conteúdo Web, denominada de WCAG, visando garantir o avanço de interfaces interoperáveis e a acessibilidade na web. A WCAG, utilizada por governos e empresas, está organizada em quatro princípios, de forma que para ser acessível, a plataforma deve ser perceptível, operável, compreensível e robusta (W3C, 2014).

Já o eMAG, atualmente na sua versão 3.1, publicada em abril de 2014, é um documento que contém um conjunto de recomendações para garantir a acessibilidade em sites e portais do governo brasileiro. Com sua primeira versão publicada em 2005, teve em 2007, através da Portaria $n^{\circ} 3$ de 7 de maio, a institucionalização, tornando obrigatória a sua observância pelos órgãos públicos (eMAG, 2007). O modelo apresenta algumas seções, que auxiliam desenvolvedores e facilitaram o desenvolvimento da pesquisa.

As seções foram numeradas e distribuídas em: 1. marcação, 2. comportamento, 3. conteúdo/informação, 4. apresentação/design, 5. multimídia, 6. formulário. Dessa forma, diferente da WCAG, a divisão se dá por área e não por nível de prioridade. No eMAG, há uma seção específica para multimídia, de forma que se o site ou portal web apresentar algum conteúdo em vídeo, por exemplo, deve seguir todas as recomendações determinadas para esse conteúdo (eMAG, 2007).

\subsection{MOOC}

Massive Open Online Course é uma expressão que segundo Grainger (2013, p. 2) foi "criada por Dave Cormier e Bryan Alexander em 2008, para descrever um 
modelo de curso online desenvolvido pelos acadêmicos Stephen Downes e George Siemens". De acordo com Dias Júnior (2017, p. 10) "o surgimento dos MOOCs contribuiu para fortalecer as mudanças nos paradigmas educacionais existentes, além de vir ao encontro do processo de democratização da educação e dos anseios do novo perfil de aluno da era digital, cada vez mais presente nas instituições de ensino.

Dessa forma, dentre as características principais dos MOOCs, estão a importância de serem abertos, ou seja, disponíveis na internet e sem custos; participativo, permitindo a troca de experiência e contribuições entre os usuários; e distribuído, visando atingir uma rede de participantes ilimitada (Baturay, 2015). A análise de MOOCs é tema de estudos recentes, Kunt, Ulbricht e Fadel (2015, p. 1) desenvolveram uma "validação de acessibilidade: um estudo preliminar em plataformas MOOC". Já Nascimento (2019, p. 8), apresentou em sua dissertação sobre "Acessibilidade e usabilidade em plataformas MOOC: indicador de boas práticas". Por fim, Klaus et al (2016, p. 120) desenvolveu o trabalho intitulado de "Acessibilidade em cursos abertos e massivos".

Os conceitos apresentados acima, bem como as orientações e recomendações dos modelos, serviram de base para a definição da metodologia implantada na execução da pesquisa para identificar o grau de acessibilidade nos MOOCs.

\section{Metodologia}

A pesquisa realizada é classificada como exploratória, tendo em vista que busca informações sobre acessibilidade em MOOCs previamente definidos, identificando as particularidades das plataformas estudadas Severino (2007). Para a realização do trabalho, as atividades foram divididas em 5 etapas, dependentes entre si, conforme imagem abaixo.

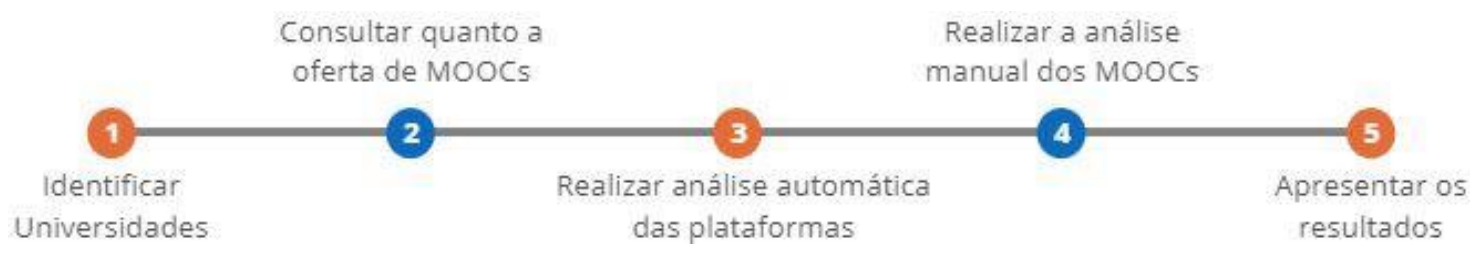

Figura 2 - fases da pesquisa

A primeira etapa consistiu na identificação de todas as universidades federais do Brasil, no site do Ministério da Educação - MEC, através do Cadastro Nacional de Cursos e Instituições de Educação Superior Cadastro e-MEC. Para a busca, foi definida o tipo de "Consulta avançada" e selecionado no filtro apenas os campos "Pública Federal" e "Universidade", possibilitando assim gerar a lista somente com as instituições que fizeram parte do escopo do desenvolvimento do trabalho.

De posse desses dados, foi possível ingressar no Sistema Eletrônico do Serviços de Informação ao Cidadão (e-SIC), da Controladoria Geral da União (CGU) para consultar se as instituições oferecem à sociedade cursos abertos. Nessa solicitação de informação, foi questionado se a universidade oferece cursos online, no formato aberto (MOOC) para a sociedade e em caso de positivo, qual o link para o ambiente.

À partir disso, partiu-se para a etapa de avaliação das plataformas de cursos abertos de acordo com os requisitos definidos pelo W3C e pelo eMAG. Essa etapa foi automatizada, através dos validadores de acessibilidade, disponíveis em http://www.acessibilidade.gov.pt/ para $\mathrm{o} \quad \mathrm{W} 3 \mathrm{C}$ e http://asesweb.governoeletronico.gov.br para o eMAG. 
Além das ações anteriores, foi realizada uma análise manual dos ambientes de MOOC, a qual, consistiu em acessar os cursos com o objetivo de avaliar todos os cursos que possuem conteúdo multimídia em vídeo. A primeira avaliação foi quanto ao uso das diferentes plataformas e a possibilidade de realização dos cursos por elas, dessa forma, foram utilizados um notebook e um dispositivo móvel (smartphone) para essa análise.

Ainda na etapa quatro, houve a identificação de todos os cursos que continham material multimídia, mais especificamente vídeos, os quais foram analisados seguindo os requisitos de acessibilidade: possibilidade de controlar a apresentação através do teclado (quando de uso de notebook), ou seja, que o usuário possa iniciar, parar, avançar e retroceder o vídeo de acordo com sua necessidade, somente utilizando teclas de atalho; utilização de audiodescrição para descrever o que está sendo apresentado em tela; uso da Linguagem Brasileira de Sinais (LIBRAS), para que o conteúdo possa ser entendido por pessoas com dificuldade ou ausência de audição; e por fim, uso de legendas, facilitando o entendimento do conteúdo que está sendo falado.

Por fim, os dados obtidos foram descritos e tabulados, visando apresentar de forma intuitiva o grau de acessibilidade presente nos MOOCs avaliados. Além disso, foram analisados os dados de diferentes autores, visando contextualizar com os resultados obtidos nessa pesquisa, os quais, são apresentados na sequência.

\section{Resultados}

A consulta realizada ao site do Ministério da Educação, retornou com a lista de 63 Universidades, ao passo, que todas elas foram questionadas quanto à disponibilização de MOOCs. A consulta, realizada via e-SIC, tem como prazo limite para resposta 30 dias, sendo que 61 instituições responderam dentro do prazo estipulado pela Controladoria Geral da União.

Dentre as respondentes, 14 informaram disponibilizar cursos abertos para a sociedade, são elas: Universidade Federal do Rio Grande (FURG), Universidade Federal Fluminense (UFF), Universidade Federal de Goiás (UFG), Universidade Federal de Mato Grosso (UFMT), Universidade Federal do Recôncavo da Bahia (UFRB), Universidade Federal do Rio Grande do Sul (UFRGS), Universidade Federal do Rio Grande do Norte (UFRN), Universidade Federal de Santa Catarina (UFSC), Universidade Federal de São Carlos (UFSCar), Universidade Federal de Santa Maria (UFSM), Universidade Federal de Viçosa (UFV), Universidade Federal de Itajubá (UNIFEI) e Universidade Federal da Lusofonia Afro-Brasileira (UNILAB).

À partir disso, iniciou-se as etapas de análise, as quais, consistiram na identificação e classificação das plataformas de cursos abertos, quanto aos requisitos do W3C, site http://www.acessibilidade.gov.pt/ e do eMAG, site http://asesweb.governoeletronico.gov.br, onde foram utilizados os links das plataformas de cursos abertos informados pelas universidades e gerados os resultados apresentados no gráfico abaixo: 


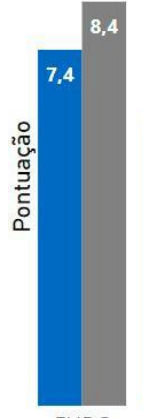

FURG

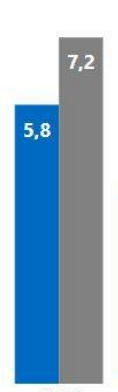

UFF
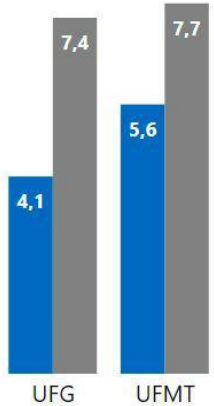
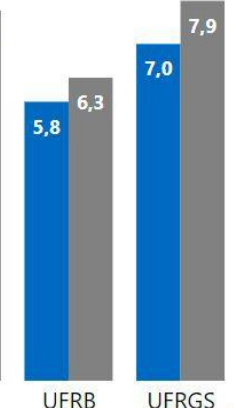

UFRGS

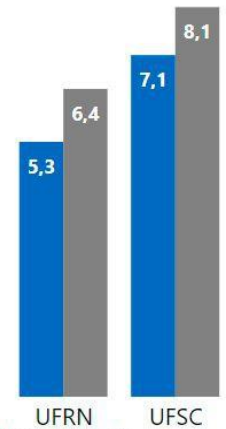

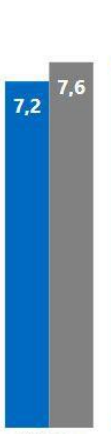

UFSCar UFSM

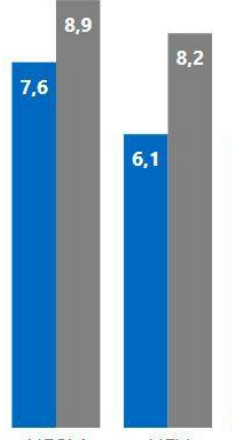

UFV
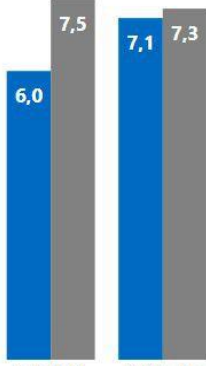

UNIFEI UNILAB

Figura 3 - gráfico de pontuação dos requisitos do W3C e eMAG.

Através da análise, foi possível verificar que os índices de atendimento ao eMAG foram superiores ao índice do $\mathrm{W} 3 \mathrm{C}$ em todas as plataformas avaliadas. Ainda assim, identificou-se que com poucas exceções, os números mostram-se insuficientes partindo da premissa de atendimento integral dos requisitos.

$\mathrm{Na}$ etapa seguinte, de análise manual, foi avaliado se os conteúdos multimídias em vídeo possuem audiodescrição, legendas e Lingua Brasileira de Sinais (LIBRAS), além de identificar se o vídeo pode ser totalmente controlado pelo usuário através do teclado, possibilitando iniciar, pausar, reiniciar ou parar a apresentação quando desejar. As avaliações foram realizadas utilizando um notebook e um smartphone analisando também as possibilidades de realização dos cursos em diferentes dispositivos. Os resultados estão descritos abaixo:

FURG: Apesar da instituição ter informado que os cursos são livres e abertos, para o acesso foi exigido e-mail institucional da FURG, inviabilizando o cadastro. Dessa forma, apesar da navegação no site ter sido possível em todos os dispositivos usados, não foi possível realizar a avaliação específica do conteúdo multimídia.

UFF: Atualmente a universidade oferece 12 cursos, sendo que 7 deles foram passíveis das análise. Independente de plataforma, foi possível acessar o conteúdo dos cursos sem dificuldade, no entanto, no que tange aos recursos multimídias, foram identificados 3 diferentes players de vídeo. Em dois deles foi possível utilizar o teclado para controlar a apresentação, no outro, esse controle mostrou-se limitado. Quanto aos recursos, nenhum dos conteúdos apresentou audiodescrição e LIBRAS. A maioria apresentou como língua o inglês, sem legenda em português. Por fim, o curso que a língua utilizada foi o português, acompanhava somente legenda em inglês.

UFG: Apesar da vasta quantidade de cursos disponíveis e de possibilitar o acesso tanto no notebook quanto no smartphone, somente dois cursos foram acessíveis para usuário externos à instituição, no entanto, nenhum dos curso continha material multimídia para avaliação, impossibilitando de realizar a análise proposta na pesquisa.

UFMT: A plataforma oferece atualmente 20 cursos, acessíveis sem dificuldade em dispositivos móveis. A universidade utiliza o youtube para armazenar os vídeos, de forma que possibilita o controle da apresentação através do teclado. No entanto, não foi identificado audiodescrição e linguagem de sinais nos conteúdos, somente áudio em português e legendas automáticas do youtube, também em português.

UFRB: Acessível em todos os dispositivos utilizado para a análise, a UFRB oferece em seu ambiente 12 cursos abertos. Desses, 10 cursos possibilitaram a inscrição e avaliação, sendo que nenhum deles apresentou LIBRAS e audiodescrição. Ao utilizar o 
player do youtube em todos os cursos, foi possível utilizar atalhos do teclado para controle de apresentação. Todos os cursos são ministrados em português, com possibilidade de legenda automática do youtube, também em português, em 9 deles.

UFRGS: Com 49 cursos ofertados, a plataforma da UFRGS mostrou-se de fácil acesso tanto via notebook quanto via smartphone. Foram avaliados conteúdos dos 49 cursos disponíveis, sendo que o curso "Desconstruindo o racismo na prática" apresentou todos os itens de acessibilidade avaliados, com idioma em português, possui audiodescrição e legendas (automáticas do youtube). Os demais cursos, não apresentaram conteúdo com audiodescrição, porém, com exceção de um, que não utiliza o player do youtube, todos possibilitaram a inclusão de legendas. Por fim, um dos cursos, seguindo a sua proposta, apresenta idioma de áudio e legenda em inglês.

UFRN: O ambiente avaliado da UFRN, apresenta nos filtros de busca de cursos os que possuem audiodescrição e legenda, no entanto, não possível acessá-los para identificar a presença dos requisitos de acessibilidade pois os cursos não atendem o formato aberto, sendo cursos pagos. Dessa forma, a avaliação quanto aos cursos da UFRN ficou incompleta.

UFSC: Com 462 cursos ofertados no total, a UFSC disponibiliza 164 que podem ser acessados de forma livre. No entanto, não são cursos produzidos para atendimento à sociedade de acordo com a proposta dos MOOCs, mas sim, disciplinas ministradas em seus cursos, que ficam disponíveis para acesso externo. Esses cursos apresentam, em sua maioria, documentos textuais e fotos, o que dificultou a análise proposta para essa pesquisa, no entanto, foram identificados 2 cursos com vídeos, sendo $1 \mathrm{com}$ idioma de áudio em inglês e legenda em português e 1 com áudio em português, mas sem legendas e LIBRAS. Os cursos possibilitaram o controle dos vídeos pelo teclado e foram acessados do celular sem maiores dificuldades.

UFSCar: De 28 cursos avaliados, somente 2 apresentaram conteúdo em vídeo, os quais, não contemplam audiodescrição e legenda. Atendem as questões relacionadas ao acesso via smartphone e controle via teclas de atalhos, além de possuirem seu idioma de áudio em português. Nenhum dos curso apresentou língua de sinais.

UFSM: Os cursos são ofertados por períodos, como nenhum curso estava com inscrições abertas, não foi possível avaliar a acessibilidade em conteúdo multimídia da Instituição.

UFV: A UFV utiliza a plataforma chamada de "UAITEC", do Governo do Estado de Minas Gerais, que atualmente oferece 68 cursos abertos. No entanto, ao tentar realizar as inscrições nos cursos, ocorreu um erro no site, impossibilitando a conclusão do processo. Dessa forma, a pesquisa não pode ser realizada no ambiente.

UNIFEI: São ofertados 42 cursos no formato aberto pela UNIFEI, foram avaliados 5 cursos que possuíam vídeos para capacitação da sociedade, os quais, não apresentaram LIBRAS e audiodescrição. Assim como a maioria dos demais avaliados, a plataforma da UNIFEI também utiliza o youtube como forma de disponibilização dos vídeos, o que permitiu a inclusão de legendas automáticas em português. Com áudio também em português, todos os vídeos foram acessados pelo smartphone sem dificuldades e possibilitaram o controle via teclado, quando acessados via notebook.

UNILAB: A plataforma da UNILAB não apresenta cursos disponíveis ainda, fato este, que inviabilizou a pesquisa.

Após a conclusão da análise de todos os MOOCs informados pelas universidades, constatou-se que apenas na UFF, UFMT, UFRB, UFRGS, UFSC, UFSCar e UNIFEI a plataforma informada possibilitou a realização das análises necessárias para o atingimento do objetivo do artigo, conforme escopo definido. Dessa 
forma, a tabela abaixo sintetiza os resultados da avaliação do grau de acessibilidade encontrado nos vídeos disponibilizados nos MOOCs.

Tabela 1 - resultado sintetizado da pesquisa realizada nos vídeos dos MOOCs

\begin{tabular}{l|l|l|l|l|l}
\hline Universidade & Multiplataforma & Legenda & Libras & Audiodescrição & Atalhos teclado \\
\hline UFF & Sim & $0 \%$ & $0 \%$ & $0 \%$ & $67 \%$ \\
\hline UFMT & Sim & $100 \%$ & $0 \%$ & $0 \%$ & $100 \%$ \\
\hline UFRB & Sim & $90 \%$ & $0 \%$ & $0 \%$ & $100 \%$ \\
\hline UFRGS & Sim & $100 \%$ & $2 \%$ & $2 \%$ & $100 \%$ \\
\hline UFSC & Sim & $50 \%$ & $0 \%$ & $0 \%$ & $100 \%$ \\
\hline UFSCar & Sim & $0 \%$ & $0 \%$ & $0 \%$ & $100 \%$ \\
\hline UNIFEI & Sim & $100 \%$ & $0 \%$ & $0 \%$ & $100 \%$ \\
\hline
\end{tabular}

Considerando os resultados encontrados, foi possível perceber que há uma preocupação quanto a utilização em multiplataformas, ou seja, possibilitar que o aluno possa realizar o curso também em dispositivos móveis, através de plataformas responsivas. Além disso, o fato de muitos MOOCs utilizarem player do youtube, facilita para que haja o controle dos vídeo através de atalhos do teclado e incluir legendas, que mesmo de forma automática, possibilitam o entendimento do conteúdo sem maiores dificuldades.

No entanto, fica claro que não há uma preocupação com audiodescrição e LIBRAS nos vídeos, sendo funcionalidades percebidas em apenas um curso no MOOC da Universidade Federal do Rio Grande do Sul, que apresenta diferentes opções de visualização, conforme necessidade do aluno.

Quanto à análise automatizada, fica claro que há necessidade de melhorias nos ambientes onde os cursos são disponibilizados, tendo em vista que a maioria das notas está abaixo de 8 pontos. Estevam (2019, p. 53), após avaliar a acessibilidade em MOOCs, que não são oferecidos por universidades, diz que "lamentavelmente, mesmo com o avanço das tecnologias Web, as pessoas com deficiência visual ainda enfrentam algumas barreiras em relação ao acesso às informações disponibilizadas na Internet". Reafirma ainda que "mesmo com os erros e avisos encontrados, ainda consegue garantir o acesso de pessoas com deficiência visual" (Estevam, p. 53). Essas afirmações vão ao encontro dos resultados encontrados na análise das plataformas oferecidas pelas instituições públicas, onde constata-se a presença de alguns recursos de acessibilidade, mesmo com necessidades de melhorias.

Por outro lado, Nascimento (2019), em sua análise de usabilidade e acessibilidade em MOOCs afirma que normas estão sendo aplicadas conforme as inovações tecnológicas, visando adequar às limitações físicas, cognitivas ou tecnológicas dos usuários. Isso é possível perceber nas legislações que surgem e aprimoram-se visando garantir a inclusão digital de todos. Além disso, em relação a acessibilidade, Nascimento (2019, p. 93) afirma que "uma interface estruturada e padronizada pode minimizar as necessidades dos usuários, independente de suas limitações". Este fator foi analisado na pesquisa realizada, principalmente no que tange a responsividade das plataformas e uso nos dispositivos móveis, além da possibilidade de controlar os players de vídeo através de teclas de atalho, dois quesitos que obtiveram um retorno considerado satisfatório. 
Dessa forma, os resultados apresentados, demonstram que embora ainda seja necessário o aperfeiçoamento das plataformas, para que possam ampliar o atendimento aos diferentes tipos de acessibilidade, já há algumas ações que visam garantir às pessoas com deficiência a realização de cursos de capacitação.

\section{Considerações finais}

Esta pesquisa consistiu em analisar diferentes requisitos de acessibilidade nas plataformas MOOCs, disponibilizadas pelas universidades federais do Brasil. Após a identificação das instituições e a consulta sobre a oferta de plataformas de cursos abertos para a sociedade, foi possível fazer a análise, a qual apresentou resultados significativos no que tange a necessidade de atuar ainda mais para oferecer ambientes mais acessíveis.

Esses resultados demonstram que, embora sejam necessárias otimizações, os sites onde são disponibilizados os cursos, são responsivos e podem ser acessados inclusive de dispositivos móveis. Das 14 plataformas disponíveis, apenas 7 puderam ser testadas na integralidade ao longo da pesquisa, dessas, somente a da Universidade Federal do Rio Grande do Sul apresentou um curso que contemplou todos os itens analisados.

Portanto, diante desses resultados, ficou comprovado que faz-se necessária uma atenção especial das universidades para melhoria de seus MOOCs, os quais, são de grande importância para o processo de ensino-aprendizagem e para a capacitação da sociedade.

\section{Referências Bibliográficas}

BATURAY, Meltem Huri. An overview of the world of MOOCs. Procedia-Social and Behavioral Sciences, v. 174, p. 427-433, 2015.

BRASIL. Lei ${ }^{\circ} 10.048$ de 08 de novembro de 2000. Dá prioridade de atendimento às pessoas que especifica, e dá outras providências. Brasília. Disponível em: https://www.presidencia.gov.br/ccivil_03/Leis/L10048.htm. Acesso em: 09 out. 2019.

BRASIL. Lei $\mathrm{n}^{\mathrm{o}}$. 10.098, de 19 de Dezembro de 2000. Estabelece normas gerais e critérios básicos para a promoção da acessibilidade das pessoas portadoras de deficiência ou com mobilidade reduzida. Brasília. Disponível em: https://www.presidencia.gov.br/ccivil_03/Leis/L10098.htm. Acesso em: 09 out. 2019.

CANDEIAS, Cezar Nonato Bezerra; CARVALHO Luis Henrique Pereira de. O uso de videoaulas como ferramenta no processo de ensino e aprendizagem em química. 2016. Disponível em: https://eventos.set.edu.br/index.php/simeduc/article/view/3306. Acesso em: 29 set. 2019.

DIAS JUNIOR, José Barbosa. Meta-MOOC: Uma ferramenta para geração de MOOCs adaptativos e personalizáveis. 2017. Disponível em: https://repositorio.ufu.br/bitstream/123456789/21135/3/MetaMoocFerramenta.pdf.

Acesso em: 11 out. 2019.

E-MAG. Modelo de Acessibilidade de Governo Eletrônico. 2007. Disponível em: http://governodigital.gov.br/acoes-e-projetos/e-MAG. Acesso em: 10 out. 2019. 
ESTEVAM, Marcelo dos Santos. Avaliação de acessibilidade visual de curso na plataforma MOOC com base no eMAG. 2019. Disponível em: http://www.repositorio.ufc.br/bitstream/riufc/43461/1/TCC\%20MARCELO.pdf. Acesso em: 11 out. 2019.

FERRAZ, Reinaldo. Acessibilidade na Web. São Paulo: Ed. Senac, 2017.

GONÇALVES, Bruno Miguel Ferreira. MOOC e b-Learning: uma proposta para o mestrado em TIC na Educação e Formação do Instituto Politécnico de Bragança. Escola Superior de Educação de Bragança, 2013. Dissertação de Mestrado.

GRAINGER, Barnaby. Introduction to MOOCs: avalanche, illusion or augmentation. 2013. Disponível em: http://iite.unesco.org/pics/publications/en/files/3214722.pdf. Acesso em: 11 out. 2019.

IBGE. Censo Demográfico 2010: Características gerais da população, religião e pessoas com deficiência. Rio de Janeiro, 2012.

KUNTZ, Viviane Helena; ULBRICHT, Vania Ribas; FADEL, Luciane. Validação de acessibilidade: um estudo preliminar em plataformas MOOC (Massive Open Online Course). Blucher Design Proceedings. v. 2, n. 1, p. 1291-1301, 2015.

NASCIMENTO, Heluiza Ormelez de Almeida. Acessibilidade e Usabilidade em Plataformas MOOC: Indicadores de Boas Práticas. Dissertação de mestrado, 2019.

SEVERINO, Antonio Joaquim. Metodologia do Trabalho Científico. São Paulo: Cortez, 2007.

SCHLÜNZEN JUNIOR, Klaus et al. Acessibilidade em cursos abertos e massivos. Journal of Research in Special Educational Needs. v. 16, p. 120-125, 2016.

YUAN, Li; POWELL, Stephen. MOOCs and Open Education: Implications for Higher Education. (2013). Disponível em: https://www.researchgate.net/publication/265297666_MOOCs_and_Open_Education_I mplications_for_Higher_Education. Acesso em: 3 out. 2019.

W3C. Web Content Accessibility Guidelines (WCAG) 2.1. 2018. Disponível em: https://www.w3.org/TR/WCAG21/. Acesso em: 06 out. 2019.

W3C BRASIL. Cartilha Acessibilidade na Web. Disponível em: https://www.w3c.br/. Acesso em: 06 out. 2019. 